\title{
The Electrical Conductivity of Fertilized and Unfertilized Eggs.
}

By

\author{
J. Gray, B.A., \\ King's College, Cambridge.
}

THE following is a preliminary account of some experiments carried out this summer at Plymouth. They were undertaken with a view to the investigation of the changes, both physical and chemical, which are induced in the egg by the entrance of a spermatozoon.

No attempt is made to discuss the results in the light of current theories. regarding the phenomena of fertilization, as it is hoped that further work will make such discussions more profitable than would be the case at present.

\section{Apparatus.}

In order to determine the electrical conductivity of the eggs, a conductivity cell was used such as would fit the holder of a small hand centrifuge. The tube was about $10 \mathrm{~cm}$. long and $1 \mathrm{~cm}$. in diameter. The electrodes were two square platinum plates each possessing two equal surfaces of $25 \mathrm{sq} . \mathrm{mm}$. These were fixed about $25 \mathrm{~mm}$. apart and were carried by two silver wires which passed through glass tubes, and which were fixed through the stopper of the tube.

In some of the earlier experiments the volume of the eggs in the tube was determined by marking their level with a fine pointed grease pencil, but in all the later experiments the tube was graduated.

This form of conductivity cell has two advantages-it fits into the holder of an ordinary centrifuge, and the volume of eggs required is small. The latter point is of great importance, quite apart from the difficulty in obtaining large quantities of ripe Echinus eggs. If too many eggs are enclosed within a tube, it is not only impossible to ensure a good percentage of fertilizations by adding a small quantity of sperm, but the overcrowding of the eggs interferes very considerably with the development of the eggs subsequent to fertilization, i.e. the rate of division differs very considerably from that of similar eggs in a large bulk of water; in extreme 
cases the development ceases at an early stage. In all my experiments, except where specially mentioned to the contrary, the eggs developed normally, although in some cases more slowly than the controls.

The temperature at which the experiments were made never differed much from room temperature, so that it was found possible to keep the temperature of the eggs constant to within $\frac{x^{\circ}}{10}$ centigrade by means of a simple thermostat. The whole apparatus stood in a large earthenware bowl containing water at room temperature.

The alternating current was obtained from a 2-volt accumulator connected to a small induction coil of high frequency. The resistance was measured by means of a Kohlrausch bridge (half a metre in length), and a telephone. The electrodes were platinized in the usual way by means of platinic chloride with a trace of lead acetate. The induction coil was placed outside the room in which the experiments were made, and by keeping the electrodes well platinized it was possible to obtain quite distinct minimal points with an ordinary telephone.

\section{Methods.}

The procedure adopted during the whole of the experiments was as follows: The ovaries of a perfectly ripe female were shaken in one or more finger-bowls containing "outside" water. The ovaries were removed after five or ten minutes, and the sea-water containing the eggs filtered through a suitable piece of bolting silk. In this way any loose pieces of ovarian tissue were removed from the eggs. The latter were now allowed to settle to the bottom of the bowl. The ripe eggs settled somewhat slowly, but after a short time sufficient eggs for one experiment could be drawn off in a clean pipette; thence they were transferred to the conductivity tube. The requisite amount of eggs having been so obtained, the tube was filled up with clean sea-water, corked and allowed to stand in a bowl of sea-water until the eggs had again settled sufficiently for the bulk of the sea-water to be removed. This having been done, the eggs were again washed in clean sea-water. After two or three such washings all the small fragments of tissue smaller than the eggs were removed, and the tube contained nothing but ripe eggs in clean seawater. After washing in this way the eggs settled somewhat more readily than when removed from the ovary, owing to the removal of the gelatinous ovarian membranes. The conductivity tube containing the eggs was then transferred to the thermostat and left until the eggs had settled to a definite volume which could be estimated without any difficulty. Great care was used to ensure that the eggs settled uniformly 
in the tube; if this precaution is not observed it will be found that repeated estimations of the resistance of the same eggs occupying the same volume give very variable results. It was found possible, however, to collect the eggs in such a way as to obtain uniform readings from repeated observations. To ensure an equal distribution of the eggs is a matter of great difficulty in the case of experiments dealing with resistances below $20 \mathrm{ohms}$; above this, however, the difficulty can be overcome by patience and repeated washings of the eggs.

The volume of the eggs having been accurately determined by means of the graduation on the tube, some of the sea-water was removed from the tube and the electrodes placed in position and the resistance of the eggs determined.

The electrodes were then removed and the tube filled with fresh seawater (care being taken not to remove any eggs with the electrodes).* One or two drops of a dilute emulsion of sperm were then added and the tube inverted so as to distribute the eggs equally through the sea-water.

After a minute the tube was allowed to stand in the water of the thermostat, until the eggs had again settled to the bottom of the tube. It was usually found that the fertilized eggs occupied a larger volume than the same eggs unfertilized; in such cases the tube was transferred to a hand centrifuge and very gently centrifuged to the required volume. The excess of sea-water was again drawn off and the electrodes placed in position and the resistance of the egg determined. The use of the centrifuge was avoided as far as possible for fear of injuring the eggs; it was found, however, that gentle use of the machine had no effect on the resistance of unfertilized eggs, or upon the subsequent development of fertilized eggs. If, however, the unfertilized eggs were so compacted as to require rather vigorous use of the centrifuge, the experiment was discontinued after the estimation of the resistance of the fertilized eggs. Such eggs were usually found to be crushed although not broken, and when returned to abundant sea-water the large majority developed normally. $\dagger$

After each estimation of the resistance of the eggs, the conductivity tube was filled with fresh sea-water and immersed horizontally in seawater whose temperature never differed from that of the thermostat by more than $1^{\circ} \mathrm{C}$.

Objections may be raised against the conclusion that the observed differences in resistance of eggs at different stages of development are

* When not actually in use the electrodes were kept immersed in clean sea-water.

+ These remarks apply to eggs which gave a resistance of $70 \mathrm{ohms}$ and upwards. 
due to the direct effects of fertilization. The following alternative suggestions might be offered :-

1. That the changes are due to the injurious effect of the current upon the eggs. For this, however, evidence is entirely unavailable. Eggs upon which definite and prolonged experiments had been made on June 18th were transferred to a bowl of clean sea-water, all the eggs divided normally, and all gave healthy larvæ. Some of these early plutei were placed in a sterilized jar and fed with a pure diatom culture ; on July 11th the plutei were large and healthy. They had developed at a normal rate, and a definite Echinus rudiment was visible. Several such observations of the development of eggs whose resistance had been measured were kept, and in each case the development was perfectly healthy and normal.* It is therefore, I think, safe to conclude that the eggs were unaffected by the passage of the current used in these experiments.

2. That the changes are due to experimental error in returning the eggs to the same volume. To determine the degree of error due to such a source, I made successive determinations on the resistance of the same lot of unfertilized eggs. I found that the variation of the readings never exceeded $2 \%$ of the total resistance (in many cases successive readings were identical). Now such a difference might perhaps explain experiments in which the total resistance is below $20 \mathrm{ohms}$, but is quite inadequate for the much larger differences which were regularly observed for eggs compacted to give higher resistances.

3. That the decrease in resistance of the eggs subsequent to fertilization is due to the presence of the fertilization membrane and not to the substance of the egg itself. It cannot, however, be suggested that the spaces between the egg are enlarged by the membrane, for the unfertilized eggs are not crushed during the experiments, and any crushing undergone by the fertilized eggs is at the expense of the membrane and not of the egg. If the eggs are closely compacted after fertilization it is almost invariably found that either the membranes are much wrinkled or are removed entirely from the egg on the addition of fresh sea-water. Again, during the course of the experiments it was found that the fertilization membranes in some batches of eggs were never pushed far out from the egg, but

* In the case of Echinus miliaris plutei, which according to Shearer, De Morgan and Fuchs fail to develop their green pigment if unhealthy, my cultures invariably possessed this character and were, in the opinion of Dr. Shearer, perfectly healthy. (Most of the cultures were discarded as soon as the Echinus rudiment had reached considerable size, but in two cultures which were preserved the larvæ underwent perfectly typical metamorphosis-a little more than a month after fertilization, which is in agreement with the rate of development of the egg under normal conditions.) 
remained rather closely applied to the egg-surface; in such cases the resistance of the fertilized eggs was, as in other cases, markedly lower than that of the unfertilized eggs. It was also found that eggs from which the fertilization membrane had been forcibly removed developed quite normally. Now Loeb has shown that the fertilization membrane is permeable to electrolytes, and I therefore conclude from the above facts that the presence of a membrane round the eggs is equivalent to a similar quantity of sea-water, and that when the eggs are reduced to the same volume as before fertilization, the distance between the eggs is identical.

The fact that after the initial fall in resistance of the eggs after fertilization there is a definite and well-marked rise in resistance, eliminates the suggestion that the changes observed are due to the presence of a fertilization membrane. Again, in a few cases (i.e. in experiments dealing with high resistances) a considerable number of the membranes are removed from the egg whilst determining the resistance of the fertilized eggs, so that when sea-water is added the membranes are found floating in it. If the eggs are not allowed to settle at once they are found still to have a resistance equal to the value obtained by the first determination after fertilization.

4. That the observed differences are due to the existence of free spermatozoa between the eggs. This is not the case, because (1) the addition of as much sperm as was used in any of these experiments does not alter the conductivity of a bulk of water equal to that of the eggs; (2) the conductivity of a concentrated emulsion of sperm is considerably lower than that of pure sea-water.

(NотE.-McClendon states that by repeatedly washing the unfertilized eggs of Arbacia he was able to prevent the formation of a fertilization membrane when the sperm entered the egg. I have never observed this in the eggs of Echinus, but prolonged washing tends to prevent the membranes being pushed out to their normal extent.) 
TABLE I.

EXPERIMENTS WITH Echinus acutus.

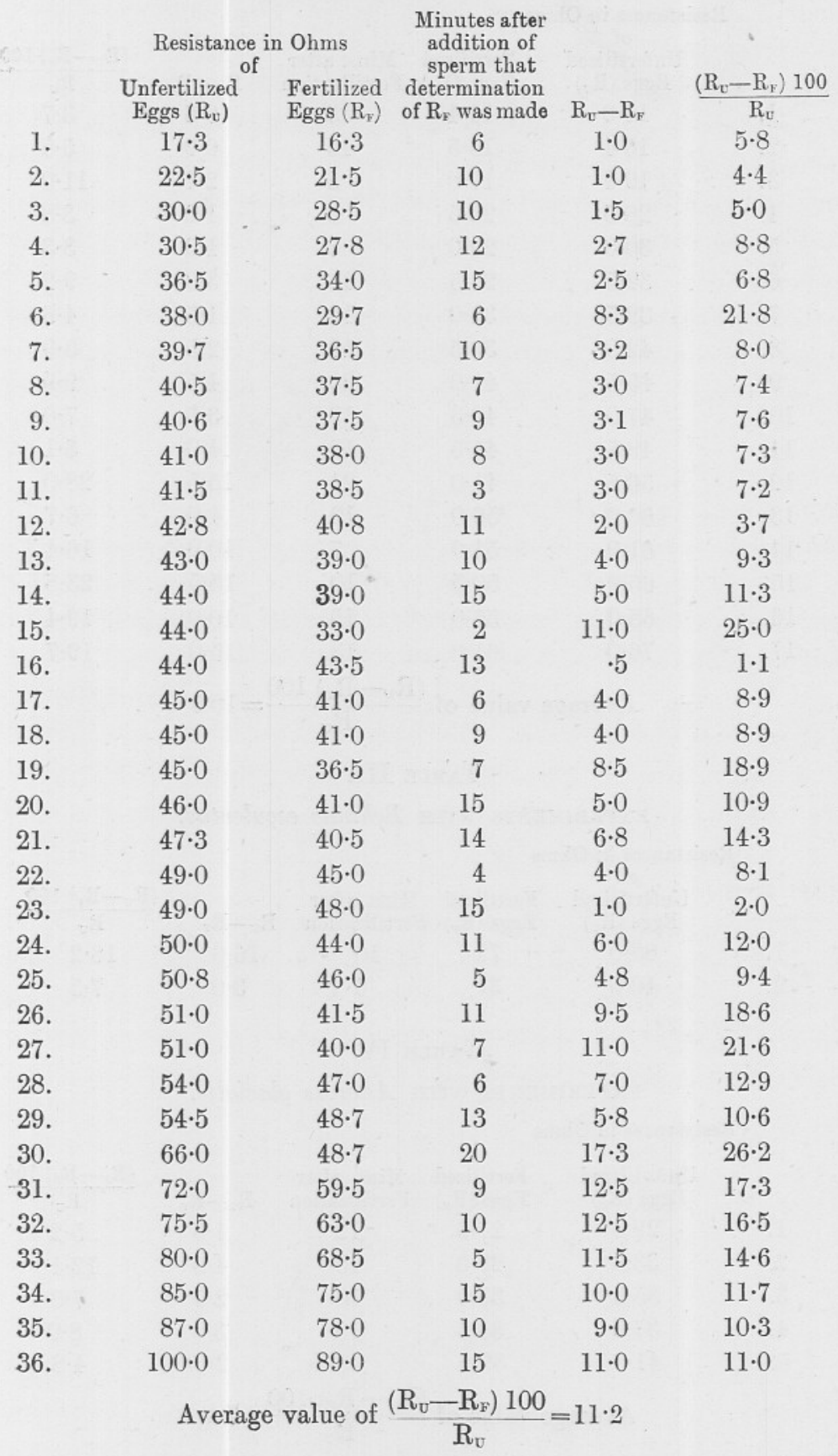


Table II.

EXPERIMENTS WITH Echinus miliaris

Resistances in Ohms

$$
\text { of }
$$

$$
\begin{gathered}
\text { Unfertilized } \\
\text { Eggs }\left(\mathrm{R}_{\mathrm{v}}\right)
\end{gathered}
$$

Fertilized Mins. after

$16 \cdot 0$

Eggs $\left(R_{F}\right)$ Fertilization

$$
\begin{array}{cc}
\mathrm{R}_{\mathrm{U}}-\mathrm{R}_{\mathrm{Y}} & \frac{\left(\mathrm{R}_{\mathrm{U}}-\mathrm{R}_{\mathrm{F}}\right) 100}{\mathrm{R}_{\mathrm{U}}} \\
0.6 & 3 \cdot 7
\end{array}
$$

1.

2.

$16 \cdot 4$

$15 \cdot 4$ 15

$15 \cdot 5$

15

0.9

$5 \cdot 5$

3. $\quad 19 \cdot 1$

$17 \cdot 0$

$2 \cdot 1$

$11 \cdot 0$

4.

$26 \cdot 5$

$25 \cdot 5$

$1 \cdot 0$

$3 \cdot 8$

5.

$30 \cdot 0$

$29 \cdot 0$

$1 \cdot 0$

$3 \cdot 3$.

$29 \cdot 5$

$3 \cdot 0$

$9 \cdot 2$

32.5

$31 \cdot 0$

1.5

$4 \cdot 6$

$32 \cdot 5$

$39 \cdot 5$

2.5

$5 \cdot 9$

$45 \cdot 5$

$41 \cdot 0$

4.5

$9 \cdot 9$

10.

$47 \cdot 0$

$40 \cdot 3$

$3 \cdot 3$

$7 \cdot 0$

$45 \cdot 5$

$4 \cdot 0$

$8 \cdot 1$

$41 \cdot 0$

$15 \cdot 5$

$28 \cdot 0$

$56 \cdot 5$

$56 \cdot 0$

21

$4 \cdot 0$

$6 \cdot 7$

$60 \cdot 0$

$51 \cdot 0$

$10 \cdot 0$

$16 \cdot 4$

15.

$65 \cdot 0$

$50 \cdot 5$

$15 \cdot 5$

$23 \cdot 8$

16.

$65 \cdot 0$

$55 \cdot 0$

$10 \cdot 0$

$19 \cdot 4$

17.

$76 \cdot 0$

$61 \cdot 0$

$15 \cdot 0$

$19 \cdot 7$

Average value of $\frac{\left(R_{U}-R_{F}\right) 100}{R_{U}}=10.9$

Table III.

EXPERIMENTS WITH Echinus esculentus.

\begin{tabular}{|c|c|c|c|c|}
\hline $\begin{array}{l}\text { Unfertilized } \\
\text { Eggs }\left(\mathrm{R}_{\mathrm{U}}\right)\end{array}$ & $\begin{array}{l}\text { Fertilized } \\
\text { Eggs }\left(R_{F}\right)\end{array}$ & $\begin{array}{l}\text { Mins. after } \\
\text { Fertilization }\end{array}$ & $\mathrm{R}_{\mathrm{U}}-\mathrm{R}_{\mathrm{F}}$ & $\frac{\left(\mathbf{R}_{V}-\mathbf{R}_{\mathrm{F}}\right) 100}{\mathbf{R}_{\mathrm{V}}}$ \\
\hline $29 \cdot 0$ & $27 \cdot 5$ & 12 & 1.5 & $5 \cdot 2$ \\
\hline $33 \cdot 0$ & $29 \cdot 0$ & 15 & $4 \cdot 0$ & $12 \cdot 1$ \\
\hline $35 \cdot 5$ & $33 \cdot 0$ & 10 & $2 \cdot 5$ & $7 \cdot 0$ \\
\hline $37 \cdot 5$ & $34 \cdot 5$ & 15 & $3 \cdot 0$ & $8 \cdot 0$ \\
\hline 41.5 & $39 \cdot 5$ & 8 & $2 \cdot 0$ & $4 \cdot 8$ \\
\hline
\end{tabular}

Resistances in Ohms

$\begin{array}{cccccc}\text { of } & \text { Unfertilized } & \text { Fertilized } & \text { Mins. after } & & \left(\mathrm{R}_{\mathrm{V}}-\mathrm{R}_{\mathrm{F}}\right) 100 \\ \text { Eggs }\left(\mathrm{R}_{\mathrm{U}}\right) & \text { Eggs }\left(\mathrm{R}_{\mathrm{F}}\right) & \text { Fertilization } & \mathrm{R}_{\mathrm{U}}-\mathrm{R}_{\mathrm{F}} & \frac{\mathrm{R}_{\mathrm{v}}}{\text { 1. }} \\ \text { 1. } & 88 \cdot 0 & 72 & 10 & 16 \cdot 0 & 18 \cdot 2 \\ \text { 2. } & 40 \cdot 0 & 37 & 6 & 3 \cdot 0 & 7 \cdot 5\end{array}$

TABLE IV.

experiments. With Asterias glacialis.

Resistances in Ohms

Average value of $\frac{\left(R_{U}-R_{F}\right) 100}{R_{U}}=7 \cdot 4$ 


\begin{tabular}{|c|c|c|c|c|}
\hline & & $\begin{array}{l}\text { Resistance of } \\
\text { Unfertilized } \\
\text { Eggs }\end{array}$ & $\begin{array}{l}\text { Resistance of } \\
\text { Within } 15 \text { mins. } \\
\text { of Fertilization }\end{array}$ & $\begin{array}{l}\text { Fertilized Eggs } \\
15-44 \text { mins, after } \\
\text { Fertilization }\end{array}$ \\
\hline 1. & Echinus acutus & $30 \cdot 0$ & $28 \cdot 5(10)$ & $29 \cdot 5(32)$ \\
\hline 2. & , $\quad$, & $43 \cdot 0$ & $39 \cdot 0(10)$ & $43 \cdot 0(38)$ \\
\hline 3. & ," & $46 \cdot 0$ & $41 \cdot 0(15)$ & $43 \cdot 5(30)$ \\
\hline 4. & , & $38 \cdot 0$ & $29 \cdot 7(6)$ & $31 \cdot 0(27)$ \\
\hline 5. & , & $45 \cdot 5$ & $36 \cdot 5(7)$ & $37 \cdot 3(28)$ \\
\hline 6. & , & $51 \cdot 0$ & $40 \cdot 0(7)$ & $41 \cdot 5(27)$ \\
\hline 7. & , & $45 \cdot 0$ & $41 \cdot 0(9)$ & $42 \quad(26)$ \\
\hline 8. & , & $50 \cdot 8$ & $46 \cdot 0(5)$ & $51 \quad(20)$ \\
\hline 9. & ", & $49 \cdot 0$ & $45 \cdot 0(4)$ & $50 \cdot 5(15)$ \\
\hline 10. & , & $80 \cdot 0$ & $68 \cdot 5(5)$ & $80 \quad(16)$ \\
\hline 11. & ," & $54 \cdot 0$ & $47 \cdot 0(6)$ & $54 .(27)$ \\
\hline 12. & , & $72 \cdot 0$ & $59 \cdot 5(9)$ & $64 \cdot 0(27)$ \\
\hline 13. & ", & $42 \cdot 8$ & $40 \cdot 8(11)$ & $42 \cdot 8(38)$ \\
\hline 14. & , & $41 \cdot 5$ & $38 \cdot 5(3)$ & $40 \cdot 0(17)$ \\
\hline 15. & ", $\quad$, & $54 \cdot 5$ & $48 \cdot 7(13)$ & $48 \cdot 7(30)$ \\
\hline 16. & Echinus miliaris & $32 \cdot 5$ & $29 \cdot 5(18)$ & $32 \cdot 0(38)$ \\
\hline 17. & , , ", & $42 \cdot 0$ & $39 \cdot 5(10)$ & $41 \cdot 0(44)$ \\
\hline 18. & ", , ", & $65 \cdot 0$ & $50 \cdot 5(10)$ & $52 \cdot 5(28)$ \\
\hline 19. & Asterias glacialis & $37 \cdot 5$ & $34 \cdot 5(15)$ & $35 \cdot 5(27)$ \\
\hline 20. & , , " & $33 \cdot 0$ & $29 \cdot 0(15)$ & $30 \cdot 5(31)$ \\
\hline
\end{tabular}

The figures in brackets in columns 2 and 3 indicate the actual number of minutes after the addition of sperm to the eggs.

\section{Conclusions.}

From these experiments I draw the following conclusions :-

1. That the entrance of the sperm into the egg causes an increase in electrical conductivity of the egg. This condition usually attains its maximum within ten minutes of adding sperm to ripe eggs.

2. That this increase in conductivity is followed by a process which returns the conductivity of the egg to or towards that of the unfertilized egg.

The chief limitation of the method lies in the fact that in order to get a measurable change in resistance between the electrodes after fertilization, the eggs must be concentrated to give an initial resistance of about $30-40$ ohms. In order to cover the electrodes with the eggs, at least $\frac{1}{2}$ c.c. of eggs is required. The volume of sea-water which the conductivity cell can hold is about 10 c.c. ; the effect of these conditions is that when the eggs are shaken in sea-water after the determination of their resistance they 
are so crowded that they develop at a rate different from that of control eggs kept in abundance of water. The change in rate of development varies with different batches of eggs and with their degree of concentration. Hence it is impossible by this method to determine the conductivity of normal eggs (i.e. eggs developing at a normal rate) at any stated moment. As, however, the development of the eggs in the conductivity tube is only abnormal in respect to time (i.e. the cleavage divisions are quite normal but occur at a varying time after those in a control), it may be concluded that the sequence of events in the two cases is the same.

A more important limitation lies in the fact that the resistance can only be determined by concentration of the eggs. We may conclude that when the eggs are concentrated at the bottom of the tube, their development very quickly becomes delayed until the return of normal conditions; hence it is not possible to take a large number of readings during one experiment.

These two factors would account for the variation found in curves obtained from the individual experiments. For example, most of them show the same maximum and minimum points during the first hour of development, but corresponding points on different curves do not agree in point of time. Again, it is almost certain that the primary rise in conductivity due to the entrance of the sperm, lasts only for some fifteen minutes or less; if therefore it is found impossible (for technical reasons) to determine the resistance of the fertilized eggs during this period, the pronounced minimal point which exists during the first ten minutes of development is entirely lost, and the curve differs radically in appearance from the normal. Owing to the rapid rate in the rise and fall of the conductivity at the commencement of development the exact time at which the resistance is determined is of considerable importance. For example, one experiment gave the following data :-

The resistance of eggs 3 mins. after fertilization was $10.5 \mathrm{ohms}$ less

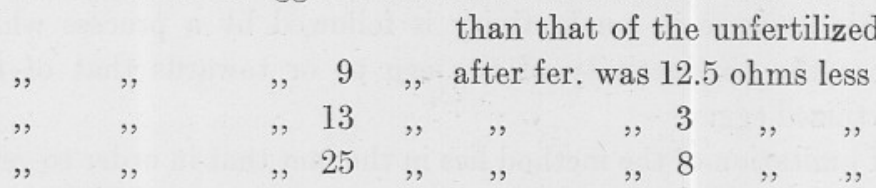

In other words, the conductivity of the eggs has undergone profound changes within 13 minutes. It is therefore necessary to discover the position of the maximum and minimum points with considerable accuracy before any quantitative value can be ascribed to the changes in conductivity which take place during the development of the normal egg. 
Without wishing to dogmatize in any way as to the explanation of these preliminary experiments, it may be pointed out that they may possibly be due to either purely physical or purely chemical changes in the eggor to a combination of such causes. The egg in the unfertilized state is remarkably impermeable to electrolytes, and can almost certainly be regarded as being enclosed within a semi-permeable membrane. In its unstimulated condition, this surface must necessarily be polarized. If now this polarization be destroyed, the membrane must become more permeable to ions than before. On this view the entrance of the sperm effects the depolarization of the plasma-membrane. After about fifteen minutes this membrane must become polarized again, but the mechanism whereby this is effected is not at present clear.

On the other hand, if the sperm carries an enzyme into the egg, which acts on some constituent of the egg-cytoplasm, then a rise in conductivity may occur as the result of the liberations of ions from unionized substances; while a reversal of this reaction will have an opposite effect.

It is hoped that further work will produce sufficient evidence to show which of these two possibilities is the more probable explanation of the experimental data, and a discussion of the results obtained by other workers is therefore postponed.

The expenses of this work have been partly defrayed by a grant from the Government Grant Committee of the Royal Society. 